\title{
Effectiveness and safety of dexamethasone implants for post-surgical macular oedema including Irvine-Gass syndrome: the EPISODIC study
}

\author{
David Bellocq, ${ }^{1,2}$ Jean-François Korobelnik, ${ }^{3,4,5}$ Carole Burillon, ${ }^{6,7}$ \\ Nicolas Voirin, ${ }^{8,9,10,11}$ Corinne Dot, $^{12}$ Eric Souied, ${ }^{13}$ John Conrath, ${ }^{14}$ \\ Solange Milazzo, ${ }^{15,16}$ Pascale Massin, ${ }^{17}$ Stéphanie Baillif, ${ }^{18}$ Laurent Kodjikian ${ }^{1,2}$
}

\begin{abstract}
Aim To assess the effectiveness and safety of intravitreal dexamethasone implants for treating post-surgical macular oedema, including Irvine-Gass syndrome refractory to first-line treatments.

Methods Descriptive, observational, retrospective, consecutive, uncontrolled, multicentre, national case series. 50 patients were included in the study between March 2011 and June 2013 with a minimum 6 months follow-up. At baseline, each patient received a dexamethasone implant $0.7 \mathrm{mg}$ (Ozurdex). Best-corrected visual acuity (BCVA), central subfield macular thickness (CSMT), and intraocular pressure (IOP) were measured at baseline and then monthly. The main outcome measure was the mean change in BCVA (in ETDRS letters (Early Treatment Diabetic Retinopathy Study): L)
\end{abstract}

Results Baseline mean \pm SD BCVA was $55.7 \pm 15.4 \mathrm{~L}$. At month 2, BCVA was $71.8 \pm 10.5 \mathrm{~L}$ and $61.2 \%$ of patients had an increase of more than 15 letters. Baseline mean CSMT was $544 \pm 117.2 \mu \mathrm{m}$ and this decreased to $302 \mu \mathrm{m}$ at month 2. Anatomic and functional recurrences were both first detected from month 3 and continued throughout follow-up, with values consistently above baseline. The peak in IOP was reached in month 1 with mean IOP of $15.3 \pm 4.6 \mathrm{~mm} \mathrm{Hg}$. Of the $39 / 50$ patients followed up for 12 months, $49 \%$ received a second injection. The anatomic and functional response and safety patterns were similar to that obtained with the first intravitreal injection, demonstrating Ozurdex's reproducibility. However, more than half of the patients followed-up for at least 1 year presented neither functional nor anatomical recurrence.

Conclusions Ozurdex would appear to be an interesting alternative therapy for treating post-surgical macular oedema, including Irvine-Gass syndrome refractory to first-line treatments.

\section{INTRODUCTION}

Irvine-Gass (IG) syndrome describes a macular oedema that develops following ophthalmic surgery. It was first clinically described in 1953 by Irvine ${ }^{1}$ in his Proctor lecture. Gass and Norton subsequently published an angiographic study of its characteristics in $1966 .^{2}$ Maumenee was the first to name it the IG syndrome. The most likely physiopathological hypothesis is that there is an inflammatory response instigated by inflammatory mediators released during and after surgical procedures, causing alterations to the blood-retinal barrier.
With improvements in cataract surgery, notably the considerable reduction in the size of the incisions required during phacoemulsification, clinically significant cystoid macular oedema (CME) with reduced visual acuity and metamorphopsia only occurs in 1$2 \%{ }^{3}$ of patients, with peak incidence occurring on average 6 weeks after surgery. However, subclinical CME-that is, with no visual impairment-is detected in almost $30 \%$ of patients with post-surgical angiography and a further $11-41 \%$ with optical coherence tomography (OCT), despite the administration of preventive treatment. ${ }^{4}$

Incidence increases in the event of complications during surgery. The identified risk factors ${ }^{5}$ are posterior capsule rupture and vitreous loss, as well as the use of iris retractors. The presence of an epiretinal membrane, a vein occlusion, a history of uveitis or diabetes, and the use of prostaglandin eye drops also increase the incidence of pseudophakic CME.

Post-surgical macular oedema is the main cause of reduced visual acuity following ophthalmic surgery. Managing the disease has proven to be complicated and no randomised studies have been conducted to establish the best therapeutic options in the last 20 years. The most common first-line treatment is a combination of oral acetazolamide, used off label, and the topical administration of non-steroidal antiinflammatory drugs (NSAIDs), used in compliance with the approved indications.

Second-line treatments include a range of therapies used off label: intravitreal injection of steroid such as triamcinolone or dexamethasone implants, ${ }^{6-11}$ or intravitreal injection of antivascular endothelial growth factor (VEGF), ${ }^{12} 13$ or subcutaneous injections of interferon $\alpha 2 \mathrm{a} .{ }^{14}$

Ozurdex is a biodegradable intravitreal implant which delivers $0.7 \mathrm{mg}$ of the corticosteroid dexamethasone into the vitreous and the retina. The dexamethasone acts on all inflammatory mediators. The implant has been approved for the treatment of macular oedema (ME) secondary to retinal vein occlu$\operatorname{sion}^{15}$ and for non-infectious posterior uveitis. ${ }^{16}$ As IG is primarily an inflammatory response and Ozurdex has already been used to treat 27 patients with IG in a phase 2 study on uveitis, ${ }^{11}$ we believed it would be of interest to collect data on a larger number of patients. Moreover, in the study by Williams et al, ${ }^{11}$ patients with IG syndrome were unfortunately mixed in with uveitis patients.

The objective of this study was to assess the anatomic and functional EffectIveness, as well as the 
Safety Of Dexamethasone implants (Ozurdex) in treating postsurgical macular oedema including Irvine-Gass syndrome (EPISODIC study).

\section{PATIENTS AND METHODS}

A descriptive, observational, retrospective, consecutive, uncontrolled multicentre pilot case series was conducted in France from March 2011 to June 2013.

All patients received clear, detailed prior information on the treatment and expected risks and benefits. As the data were collected retrospectively and the patients' management was not modified, according to French law (n²004-806, 9 August 2004), this study did not require research ethics committee approval. It was conducted in accordance with the law on data protection ( $n^{\circ} 2004-801,6$ August 2004).

All consecutive adult patients presenting with clinical or subclinical post-surgical macular oedema, including IG syndrome, treated with intravitreal injections of dexamethasone implant $0.7 \mathrm{mg}$ (Ozurdex) and followed up for at least 6 months, were included in the study.

The patients were treatment experienced and had received at least one previous treatment of NSAID eye drops combined with oral acetazolamide. All the previous treatments administered to each patient were identified.

Patients treated with oral corticosteroids, patients with any of the contraindications for Ozurdex set out in the June 2013 marketing approval, and patients with uncontrolled diabetes with glycosylated haemoglobin $>13 \%$ were excluded. ${ }^{11}$ Low baseline visual acuity, anatomic OCT macular changes due to oedema persistence, and a long duration of macular oedema did not constitute exclusion criteria.

Each patient underwent a standardised examination at the initial visit, and at each monthly follow-up visit, with measurement of best-corrected visual acuity (BCVA) in ETDRS letters (Early Treatment Diabetic Retinopathy Study), air-puff or applanation tonometry to measure intraocular pressure (IOP), ophthalmoscopy, and spectral-domain OCT (Cirrus HD-OCT Zeiss) to measure central subfield macular thickness (CSMT) (thickness of a circular area of $1 \mathrm{~mm}$, concentric to the foveal centre). Patients who received more than one injection received the same follow-up, with a monthly examination and recording of the same clinical and OCT data.

The main objective of our study was to assess the benefit of Ozurdex in treating post-surgical macular oedema including IG syndrome, with monthly visits over a 6-month follow-up period, by measuring BCVA in ETDRS letters. Several secondary objectives were also analysed. First and foremost, we wanted to assess the anatomic effectiveness of Ozurdex by measuring CSMT each month. We then assessed the implant's tolerance, measuring local tolerance in terms of IOP, iatrogenic retinal detachment or exogenous endophthalmitis, as well as overall tolerance. We also analysed the functional effectiveness according to initial visual acuity, and searched for a significant difference in gain between patients with an initial visual acuity $\leq 50$ letters and patients with an initial acuity $>50$ letters. Finally, we assessed the anatomic and functional effectiveness of Ozurdex in cases of repeat intravitreal injections.

\section{Statistical methods}

BCVA, CSMT, and IOP were described over time using mean $\pm \mathrm{SD}$ at each time point. Baseline values were at the day of Ozurdex injection and measures were repeated for each patient at months 1, 2, 3, 4, 5, and 6. A subgroup of patients underwent a second injection, and measures were also repeated for each patient at months $1,2,3,4,5$, and 6 after this second injection.

Differences in BCVA, CSMT, and IOP at months 1, 2, 3, 4, 5, and 6 compared to baseline values were assessed using univariate analysis of variance (ANOVA), taking into account the dependence of repeated measures over time for each patient. For this, the lme function of the package nlme $\mathrm{e}^{17}$ in the software $\mathrm{R}^{18}$ was used. A value of $p<0.05$ was considered statistically significant.

\section{RESULTS}

This retrospective study was conducted in nine centres located in mainland France (see online supplementary appendix 1). A case series was constituted, with 50 consecutive patients included between March 2011 and June 2013, with a minimum follow-up period of 6 months. The population characteristics are shown in table 1.

The mean age was 72.1 years. The population was made up of more women than men (58\% women). There was no difference in terms of laterality.

In regard to the cause of macular oedema, $82 \%$ of the cases were secondary to phacoemulsification cataract surgery (IG syndrome), $6 \%$ were secondary to epiretinal membrane peeling, $10 \%$ were secondary to combined cataract surgery and membrane peeling, and $2 \%$ were secondary to vitrectomy for retinal detachment.

The mean time between surgery and the diagnosis of postsurgical macular oedema was 7.8 weeks (range 4.1-14.4). All patients were treatment experienced: $62 \%$ of patients had been treated with topical NSAIDs and oral acetazolamide, 16\% had received the same treatment associated with intravitreal injection of triamcinolone, $7 \%$ had the same treatment associated with intravitreal injection of anti-VEGF, and $8 \%$ had received at least

Table 1 Baseline patient characteristics

\begin{tabular}{|c|c|c|}
\hline & Number & $\%$ \\
\hline Mean age (range) & $72.1(51-83)$ & \\
\hline \multicolumn{3}{|l|}{ Sex } \\
\hline Male & 21 & 42 \\
\hline Female & 29 & 58 \\
\hline \multicolumn{3}{|l|}{ Laterality } \\
\hline Right & 24 & 48 \\
\hline Left & 26 & 52 \\
\hline \multicolumn{3}{|l|}{ Type of surgery } \\
\hline Phacoemulsification & 41 & 82 \\
\hline ERM peel & 3 & 6 \\
\hline Combined phacoemulsification-ERM peel & 5 & 10 \\
\hline Vitrectomy for retinal detachment & 1 & 2 \\
\hline \multicolumn{3}{|l|}{ Previous treatment } \\
\hline Acetazolamide+NSAIDs only & 31 & 62 \\
\hline Acetazolamide+NSAIDs only+triamcinolone injection & 8 & 16 \\
\hline Acetazolamide+NSAIDs only+anti-VEGF injection & 7 & 14 \\
\hline$>3$ treatments & 4 & 8 \\
\hline $\begin{array}{l}\text { Mean time from diagnosis in weeks } \\
\text { (minimum to maximum) }\end{array}$ & $7.8(4.1-14.4)$ & \\
\hline $\begin{array}{l}\text { Initial mean BCVA in letters (minimum to maximum) } \\
\text { SD }\end{array}$ & $\begin{array}{l}55.7(20-80) \\
15.4\end{array}$ & \\
\hline $\begin{array}{l}\text { Initial mean CSMT in } \mu \mathrm{m} \text { (minimum to maximum) } \\
\text { SD }\end{array}$ & $\begin{array}{l}544(296-789) \\
117.2\end{array}$ & \\
\hline
\end{tabular}

BCVA, best-corrected visual acuity; CSMT, central subfield macular thickness; ERM, epi-retinal membrane; NSAIDs, non-steroidal anti-inflammatory drugs; VEGF, vascular endothelial growth factor. 
three different treatments before their first intravitreal injection of Ozurdex.

Functional effectiveness was assessed on a monthly basis by measuring mean \pm SD BCVA using the ETDRS scale. Baseline BCVA, measured on the day of the first injection of Ozurdex' was 55.7 \pm 15.4 letters (L). At month 1 , mean BCVA was 68.3 $\pm 13.0 \mathrm{~L}$, representing a gain of $12.6 \mathrm{~L}(\mathrm{p}<0.001)$. At month 2, BCVA was $71.8 \pm 10.5 \mathrm{~L}$, a gain of $16.1 \mathrm{~L}$ (more than three lines on the ETDRS scale) $(p<0.001)$. From month 3 onwards, functional recurrence was observed with a reduction in BCVA to $67.7 \pm 13.3 \mathrm{~L}$ (a gain of $12.0 \mathrm{~L}$ from baseline) $(p<0.001)$. The drop in visual acuity continued throughout the follow-up period with BCVA of $63.1 \pm 15.5 \mathrm{~L}$ at month 4 $(p<0.001)$, then $61.9 \pm 14.0 \mathrm{~L}$ in month $5(\mathrm{p}<0.001)$, and $59.6 \pm 13.5 \mathrm{~L}$ in month $6(\mathrm{p}=0.002)$ (figure 1$)$, meaning that BCVA at 6 months was still better than baseline. The percentage of patients with an increase $>15$ letters was also assessed over the course of the follow-up period. At month 2, 61.2\% of patients had benefited from an increase of this magnitude and $43.8 \%$ at month $4(\mathrm{p}<0.001)$. Anatomic effectiveness was assessed by measuring mean CSMT using spectral-domain OCT. Initial mean \pm SD CSMT was $544 \pm 117.2 \mu \mathrm{m}$. At month 2, mean CSMT was $302 \pm 76.3 \mu \mathrm{m}$, a reduction of $242 \mu \mathrm{m}$ $(p<0.001)$. From month 3 onwards, anatomic recurrence of CME was observed with CSMT of $350 \pm 68.2 \mu \mathrm{m}$. Anatomic and functional recurrences were both first detected from month 3 and continued throughout the follow-up period, with values consistently above the baseline (figure 2).

We then analysed the tolerance of the dexamethasone implant, primarily local tolerance, by measuring mean \pm SD IOP. Before the first injection, mean IOP was $12.3 \pm 2.6 \mathrm{~mm} \mathrm{Hg}$. At month 1 , mean IOP was $15.3 \pm 4.6 \mathrm{~mm} \mathrm{Hg}$, the maximum value recorded $(\mathrm{p}<0.001)$. After this first month, mean IOP decreased consistently with a mean value of $13 \mathrm{~mm} \mathrm{Hg}$ (figure 3). We also analysed the number of patients with IOP $>25 \mathrm{~mm} \mathrm{Hg}$, which concerned three patients at the first month visit ( $6 \%$ of patients) and four patients at the second month visit ( $8 \%$ of patients). The number of patients affected then decreased; at month 3, two patients had IOP $>25 \mathrm{~mm} \mathrm{Hg}$, and at month 4 just one patient. In total, 10 patients presented ocular hypertension (IOP $>21 \mathrm{~mm} \mathrm{Hg}$ ) with mean IOP of $26 \mathrm{~mm} \mathrm{Hg}$ (range 22-33). Seven patients received one single anti-glaucoma eye drop treatment and three were treated with two eye drop treatments. No filtering surgery was required.

We then analysed the functional effectiveness according to initial visual acuity. Twenty patients had an initial visual acuity $\leq 50$

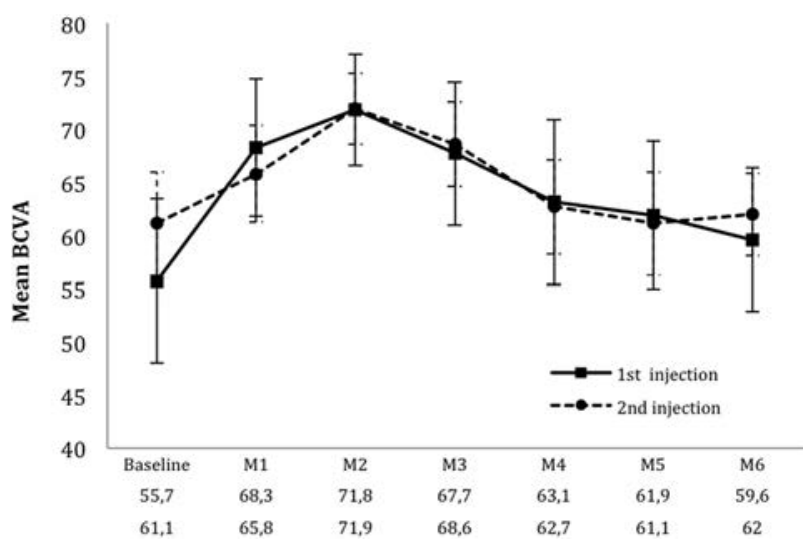

Figure 1 Functional effectiveness: changes in mean best-corrected visual acuity (BCVA) after the first two intravitreal injections of dexamethasone implant $0.7 \mathrm{mg}$ (Ozurdex). M, month.

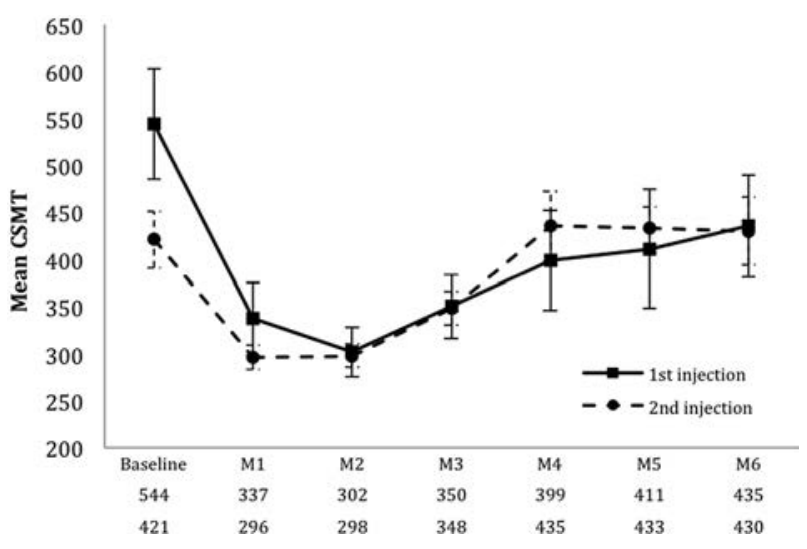

Figure 2 Anatomic effectiveness: changes in mean central subfield macular thickness (CSMT, in $\mu \mathrm{m})$ after the first two intravitreal injections of dexamethasone implant $0.7 \mathrm{mg}$ (Ozurdex). M, month.

letters (mean $40.7 \mathrm{~L}$ ) and 30 patients had initial visual acuity $>50$ letters (mean $65.8 \mathrm{~L}$ ). The mean change in visual acuity at month 2 was statistically different between the two groups, with a mean gain of $22.7 \pm 8.5 \mathrm{~L}$ at month 2 for the group ' $\leq 50$ letters' and a mean gain of $11.3 \pm 8.1 \mathrm{~L}$ at month 2 for the group ' $>50$ letters' $(\mathrm{p}<0.001)$, with BCVA at 63.4 and $77.1 \mathrm{~L}$, respectively. At month 4 , there was no statistically significant difference, with a mean gain of $9.1 \pm 11.8 \mathrm{~L}$ for the group ' $\leq 50$ letters' and $6.2 \pm 8.7 \mathrm{~L}$ for the group ' $>50$ letters' $(p=0.19)$.

Finally, we analysed the changes following the first intravitreal injection. Out of the 50 patients included in the study, 39 were followed up for at least a 12-month period. Within this cohort, 19/39 patients $(48.7 \%)$ developed functional and/or anatomic recurrence of macular oedema, meaning that more than half of the patients, followed up for at least 1 year (39 patients), only needed one injection of Ozurdex. Each of these patients was re-treated with Ozurdex. The changes in BCVA and mean CSMT were measured. The average time to re-treatment was 5.08 months (range 4.0111.2). Reproducibility was confirmed in terms of both functional and anatomic effectiveness. The day of the second intravitreal injection, mean BCVA was $61.1 \pm 9.8 \mathrm{~L}$. At the first month visit after the second intravitreal injection, mean BCVA was 65.8 \pm 9.2 L. At month 2, BCVA was $71.9 \pm 6.7 \mathrm{~L}$, representing a mean increase of $16.2 \mathrm{~L}$ compared to initial baseline $(p<0.001)$. The pattern of recurrence was similar to the pattern following the first intravitreal injection with a progressive decrease in mean BCVA throughout the follow-up period, with BCVA at $62.7 \pm 8.9 \mathrm{~L}$ at

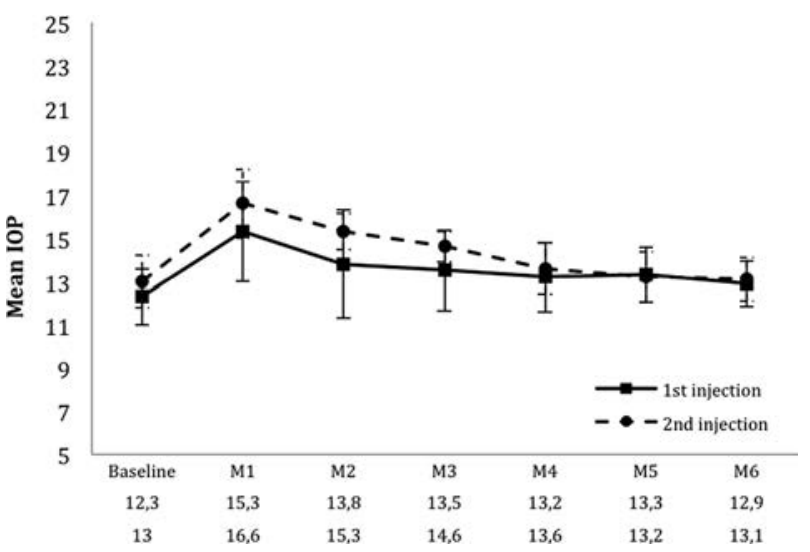

Figure 3 Tolerance: changes in mean intraocular pressure (IOP, $\mathrm{mm} \mathrm{Hg}$ ) after the two first intravitreal injections of dexamethasone implant $0.7 \mathrm{mg}$ (Ozurdex). M, month. 
month $4(p<0.001)$ (figure 1$)$. In terms of anatomic effectiveness, CSMT on the day of the second intravitreal injection was $421 \pm 60$ $\mu \mathrm{m}$. At the first monthly visit after the second injection, mean CSMTwas $296 \pm 26.6 \mu \mathrm{m}$, representing a decrease of $248 \mu \mathrm{m}$ compared to the mean baseline macular thickness $(\mathrm{p}<0.001)$. At month 2, mean CSMT was $298 \pm 24.1 \mu \mathrm{m}$, a reduction of $246 \mu \mathrm{m}$ $(\mathrm{p}<0.001)$. The pattern of recurrence was also identical, with an increase in mean CSMT from month 2 onwards, measured at 435 $\pm 74.3 \mu \mathrm{m}$ at month $4(\mathrm{p}<0.001) \quad(109 \mu \mathrm{m}$ below baseline $)$, a figure that remained consistent over the final 2 months (figure 2) $(\mathrm{p}<0.001)$.

The final parameter analysed was the tolerance after a second intravitreal injection of Ozurdex. In terms of IOP, mean pressure was $16.8 \pm 3.2 \mathrm{~mm} \mathrm{Hg}$ at the first monthly visit compared to the mean IOP before the second intravitreal injection of $13 \mathrm{~mm} \mathrm{Hg}$. IOP then progressively decreased as shown in figure 3. Three further patients presented ocular hypertension after the second intravitreal injection, with mean IOP of $22 \mathrm{~mm} \mathrm{Hg}$ (mean IOP $19 \mathrm{~mm} \mathrm{Hg}$ before the second injection). Two patients received one single pressure-lowering drug treatment and one patient received two drug treatments. No filtering surgery was required. Furthermore, there were no reported cases of retinal detachment or endophthalmitis during the study. No systemic adverse effects occurred over the course of the study.

\section{DISCUSSION}

Nowadays, patients expect an immediate benefit following cataract surgery. Post-surgical macular oedema is rare. It can heal spontaneously, but it can also persist and cause permanent damage to the macular and decrease visual acuity. IG syndrome and others kinds of post-surgical macular oedema can become a genuine therapeutic challenge, as some cases are refractory to therapy and there is no consensus on the best care management to offer patients.

As previously stated, first-line treatments often include oral acetazolamide associated with NSAID eye drops. ${ }^{19} 20$ Acetazolamide enhances the pump action of the retinal pigment epithelium by acting on carbonic anhydrase. ${ }^{21}$ The disadvantage of this treatment is the numerous adverse effects linked to acetazolamide, including renal colic, cramps, formication, and asthenia.

In the event of capsular rupture with vitreous loss and presence of vitreous in the anterior segment, the safe and thorough evacuation of vitreous from the anterior chamber, sometimes associated with a posterior vitrectomy, is the recommended firstline response.

Second-line treatments include a range of therapies used off label. Intravitreal injection of anti-VEGF can be used to inhibit VEGF released during surgical procedures. Various publications have assessed the effect of using bevacizumab or ranibizumab to treat IG syndrome, and have reported functional and anatomic benefits after several intravitreal injections. The case series conducted by Barone et al, ${ }^{12}$ composed of 10 eyes with IG syndrome, reported an increase of two lines on the ETDRS scale, after one or more intravitreal injections of bevacizumab. However, other case series have found intravitreal injection of anti-VEGF to be ineffective. The case series conducted by Spitzer et al, ${ }^{13}$ composed of 16 eyes, showed no benefit in the treatment of CME refractory to first-line treatments.

Infliximab is an anti-TNF $\alpha$ agent with immunosuppressive properties. A case series has suggested its effectiveness when administered by intravitreal injection to seven eyes with IG syndrome after the failure of intravitreal injection of anti-VEGF. ${ }^{22}$ Indeed, after an average of 2.7 intravitreal injections, mean BCVA increased from $1.14 \pm 0.59 \log$ MAR to $0.51 \pm 0.35$ $\log$ MAR $(p=0.0156)$ after 6 months' follow-up. However, adverse effects were also reported, in particular retinotoxicity. ${ }^{23}$ Another case series suggested the effectiveness of subcutaneous injections of interferon $\alpha 2 \mathrm{a}$ with an improvement in BCVA in three out of the four patients initially treated. ${ }^{14}$

The use of corticosteroids to treat post-surgical macular oedema including IG syndrome is also commonplace, often in the form of intravitreal injections of triamcinolone. Several publications have reported the effectiveness of triamcinolone in treating macular oedema secondary not only to diabetes or uveitis but also to ophthalmic surgery. ${ }^{6-10}$ The doses of triamcinolone used in the different studies vary from $2-4 \mathrm{mg}$. However, recurrence of CME is common after 6 weeks to 3 months post-injection, and the effectiveness of re-treatment varies. The case series conducted by Koutsandrea et al, ${ }^{10}$ composed of 14 eyes, demonstrated the benefit of intravitreal injections of triamcinolone for pseudophakic CME, with an improvement in visual acuity and macular thickness. Periocular corticosteroids have also been shown to be effective for pseudophakic CME refractory to topical treatments. Thach et al, in a series involving 48 patients with refractory CME, demonstrated that sub-Tenon injections improved visual acuity from 20/92 to $20 / 50(p=0.0001)$, and retrobulbar injections improved visual acuity from $20 / 97$ to $20 / 58 \quad(p=0.035)$, with no difference between the two techniques. Randazzo and Vinciguerra ${ }^{9}$ also reported improved anatomic and visual outcomes in a patient with chronic CME treated with sub-Tenon betamethasone $4 \mathrm{mg}$.

The time to recurrence of macular oedema after the first intravitreal injection of triamcinolone for IG syndrome varies between case series and occurs from the second month after the intravitreal injection onwards. ${ }^{7}$ The time to re-treatment also varies widely (2.5-24 weeks), giving a mean figure of around 8 weeks following the first intravitreal injection. ${ }^{7}$ In comparison, the mean time to recurrence after the first intravitreal injection in our case series is around 3 months and the mean time to re-treatment is much longer at 22.9 weeks. However, more than half of the patients followed-up for at least 1 year (20/39) presented neither functional nor anatomical recurrence.

Dexamethasone implants have not received specific marketing approval for the treatment of post-surgical macular oedema, including IG syndrome, although IG cases were included in the phase 2 trial. ${ }^{11}$ Ozurdex marketing approval specifies that this implant is indicated for the treatment of adult patients with inflammation of the posterior segment of the eye presenting as non-infectious uveitis. Several physiopathological models have been put forward to explain post-surgical CME and to date the most likely cause is inflammation. ${ }^{24}$ Indeed, surgery releases large quantities of inflammatory mediators such as arachidonic acid responsible for inflammatory cascade, pro-inflammatory cytokines, lysozyme or anti-VEGF. This alters the blood-retina barrier and increases vascular permeability. ${ }^{24}$ Liquid then accumulates in the external plexiform layer and inner nuclear layer, forming cystic cavities. It could therefore be suggested that postsurgical macular oedema including IG constitutes a physiopathogenic inflammation mechanism similar to posterior segment inflammation, and that using Ozurdex to treat IG could therefore be considered to constitute an approved use of the implant. Ozurdex has already been used to treat 27 patients with IG in the phase 2 case series on uveitis conducted by Williams et al. ${ }^{11}$ But patients with IG syndrome were mixed in with uveitis patients, so that no firm conclusions can be drawn regarding the IG patients. The results from this series showed that 54\% patients gained 15 letters at the third month of follow-up. The case series conducted by Dutra Meideros et $a l^{25}$ assessed the therapeutic response to Ozurdex on nine eyes with 
treatment-refractory IG syndrome, and showed an improvement in BCVA and CSMT over a 6-month follow-up period.

Our retrospective non-comparative case series included 50 consecutive patients with post-surgical macular oedema including IG syndrome in nine centres in France. The minimal follow-up was 6 months but $78 \%$ of patients were followed for 12 months. This study shows significant functional and anatomic improvements as compared to baseline, with a functional gain of more than three ETDRS lines over the follow-up period. Although some patients experienced a recurrence in CME, BCVA and/or mean CSMT at the end of follow-up were still better than at baseline.

It should also be noted that very few adverse effects were reported during follow-up. Cases of ocular hypertension were reported but were controlled with pressure-lowering medication. No filtering surgery was required. Furthermore, no cases of endophthalmitis or iatrogenic retinal detachment were reported. No systemic adverse events were observed.

In conclusion, the dexamethasone implant Ozurdex seems to be a safe and effective therapeutic option for post-surgical macular oedema including IG syndrome refractory to first-line treatments. Only half of the patients needed a second injection during the 1-year follow-up. In patients who received two injections, the effectiveness and safety of the two implants were similar. An interventional, prospective, comparative, randomised study to compare the effectiveness and safety of different second-line treatments would be helpful in confirming the results of this pilot study.

\section{Author affiliations}

${ }^{1}$ Department of Ophthalmology, Croix-Rousse University Hospital, Hospices Civils de Lyon, University of Lyon I, Lyon, France

${ }^{2}$ CNRS UMR 5510 Mateis, Villeurbanne, France

${ }^{3}$ Université de Bordeaux, ISPED, Centre INSERM U897-Epidemiologie-Biostatistique,

Bordeaux, France

${ }^{4}$ INSERM, ISPED, CHUde Bordeaux, France

${ }^{5}$ Service d'ophtalmologie, CHUde Bordeaux, France

${ }^{6}$ Department of Ophthalmology, Edouard Herriot Hospital, University of Lyon I, Lyon, France

${ }^{7}$ Institut de Biologie et Chimie des Protéines, CNRS/UMR5086, Lyon, France

${ }^{8}$ Service de Biostatistique, Hospices Civils de Lyon, Lyon, France

${ }^{9}$ Université de Lyon, Lyon, France

${ }^{10}$ Université Lyon 1, Villeurbanne, France

${ }^{11}$ CNRS, UMR 5558, Laboratoire de Biométrie et Biologie Evolutive, Equipe

Biostatistique-Santé, Villeurbanne, France

${ }^{12}$ Department of Ophthalmology, Desgenettes military Hospital, University of Val de Grâce, Lyon, France

${ }^{13}$ Hopital Intercommunal de Creteil, University Paris Est, Creteil, France

${ }^{14}$ Centre d'Ophtalmologie Monticelli-Paradis, Marseille, France

${ }^{15}$ Department of Ophthalmology, University of Picardie Jules Verne, Clinic Saint

Victor, 354, Boulevard of Beauville, Amiens, France

${ }^{16}$ Center $n^{\circ} 86$ of network EVICR-net

${ }^{17}$ Department of Ophthalmology, Assistance Publique-Hôpitaux de Paris (AP-HP)

Université Paris 7, Hôpital Lariboisière, Paris, France

${ }^{18}$ Departement of Ophthalmology, Saint Roch University Hospital, Nice Sophia Antipolis, Nice, France

Competing interests J-FK: Consultant for Alcon, Allergan, Bayer, Novartis, Thea, Roche, Zeiss. CD has sat on advisory boards for Alcon, Allergan, Bayer; lecture fees from Alcon, Alimera, Allergan, Bayer, Essilor, Novartis. ES expert for Novartis, Allergan; advisory boards for Allergan, Bayer, Novartis; lecture fees from Allergan, Heidelberg, Novartis. JC has sat on advisory boards for Alcon, Alimera, Allergan, Bayer, Novartis; lecture fees from Allergan, Bayer, Novartis. SM Investigator for trials sponsored by Alimera, Allergan, Bayer, Novartis, Leurquin and lecture fees from Alcon, Allergan, Bayer, Essilor, Novartis. PM advisory boards for Alimera, Allergan, Bayer, Novartis, Sanofi; lecture fees from Allergan, Novartis. SB: board for Novartis, Bayer, Allergan. LK Principal Investigator for trials sponsored by Novartis,
Bausch\&Lomb, Théa, Alcon; has sat on advisory boards for Alcon, Alimera, Allergan, Bayer, Bausch\&Lomb, Novartis, Théa; lecture fees from Alcon, Alimera, Allergan, Bayer, Bausch\&Lomb, Novartis, Théa.

Ethics approval As the data were collected retrospectively and the patients' management was not modified, according to French law ( ${ }^{\circ} 2004-806,9$ August 2004) research ethics committee approval was not required.

Provenance and peer review Not commissioned; externally peer reviewed.

\section{REFERENCES}

1 Irvine SR. A newly defined vitreous syndrome following cataract surgery. Am J Ophthalmol 1953;36:599-19.

2 Gass JD, Norton EW. Cystoid macular edema and papilledema following cataract extraction. A fluorescein fundoscopic and angiographic study. Arch Ophthalmol 1966;76:646-61.

3 Packer $\mathrm{M}$, Lowe J, Fine $\mathrm{H}$. Incidence of acute postoperative cystoid macular edema in clinical practice. J Cataract Refract Surg 2012;38:2108-11.

4 Perente I, Utine CA, Ozturker C, et al. Evaluation of macular changes after uncomplicated phacoemulsification surgery by optical coherence tomography. Curr Eye Res 2007:32:241-7.

5 Frost NA, Sparrow JM, Strong NP, et al. Vitreous loss in planned extracapsular cataract extraction does lead to a poorer visual outcome. Eye 1995;9:446-51.

6 Jonas JB, Sofker A. Intraocular injection of crystalline cortisone as adjunctive treatment of diabetic macular edema. Am J Ophthalmol 2001;132:425-7.

7 Benhamou N, Massin P, Haouchine B, et al. Intravitreal triamcinolone for refractory pseudophakic macular edema. Am J Ophthalmol 2003;135:246-9.

8 Thach AB, Dugel PU, Flindall RJ. A comparison of retrobulbar versus sub-Tenon's corticosteroid therapy for cystoid macular edema refractory to topical medications. Ophthalmology 1997;104:2003-8.

9 Randazzo A, Vinciguerra P. Chronic macular edema medical treatment in Irvine-Gass syndrome: case report. Eur J Ophthalmol 2010;20:462-5.

10 Koutsandrea C, Moschos MM, Brouzas D, et al. Intraocular triamcinolone acetonide for pseudophakic cystoid macular edema: optical coherence tomography and multifocal electroretinography study. Retina 2007;27:159-64.

11 Williams GA, Haller JA, Kuppermann BD, et al. Dexamethasone posterior-segment drug delivery system in the treatment of macular edema resulting from uveitis or Irvine-Gass syndrome. Am J Ophthalmol 2009;147:1048-54.

12 Barone A, Russo V, Prascina $F$, et al. Short-term safety and efficacy of intravitreal bevacizumab for pseudophakic cystoid macular edema. Retina 2009;29:33-7.

13 Spitzer MS, Ziemssen F, Yoeruek E, et al. Efficacy of intravitreal bevacizumab in treating postoperative pseudophakic cystoid macular edema. J Cataract Refract Surg 2008;34:70-5.

14 Deuter CM, Gelisken F, Stubiger N, et al. Successful treatment of chronic pseudophakic macular edema (Irvine-Gass syndrome) with interferon alpha: a report of three cases. Ocul Immunol Inflamm 2011;19:216-18.

15 Kodjikian L, Souied EH, Mimoun G, et al. EFAL Study Group. Ranibizumab versus bevacizumab for neovascular age-related macular degeneration: results from the GEFAL Noninferiority Randomized Trial. Ophthalmology 2013;120:2300-9.

16 Lowder C, Belfort RJr, Lightman S, et al. for the Ozurdex® HURON Study Group. Dexamethasone intravitreal implant for noninfectious intermediate or posterior uveitis. Arch Ophthalmol 2011;129:545-53.

17 Pinheiro J, Bates D, DebRoy S, et al. R Core Team._nlme: Linear and Nonlinear Mixed Effects Models_. R package version 3.1-117, 2014. http://CRAN.R-project. org/package $=$ nlme

18 R Core Team. R: a language and environment for statistical computing. Vienna, Austria: R Foundation for Statistical Computing, 2014. http://www.R-project.org/

19 Catier A, Tadayoni R, Massin P, et al. Advantages of acetazolamide associated with anti-inflammatory medications in postoperative treatment of macular edema. J Fr Ophtalmol 2005;28:1027-31.

20 Cox SN, Hay E, Bird AC. Treatment of chronic macular edema with acetazolamide. Arch Ophthalmol 1988;106:1190-5.

21 Marmor MF, Maack T. Enhancement of retinal adhesion and subretinal fluid resorption by acetazolamide. Invest Ophthalmol Vis Sci 1982;23:121-4.

22 Wu L, Arevalo JF, Hernandez-Bogantes E, et al. Intravitreal infliximab for refractory pseudophakic cystoid macular edema: results of the Pan-American Collaborative Retina Study Group. Int Ophthalmol 2012;32:235-43.

23 Giganti M, Beer PM, Lemanski N, et al. Adverse events after intravitreal infliximab (Remicade). Retina 2010;30:71-80.

$24 \mathrm{Xu} \mathrm{H}$, Chen M, Forrester JV, et al. Cataract surgery induces retinal proinflammatory gene expression and protein secretion. Invest Ophthalmol Vis Sci 2011;52:249.

25 Dutra Medeiros M, Navarro R, Garcia-Arumi J, et al. Dexamethasone intravitreal implant for treatment of patients with recalcitrant macular edema resulting from Irvine-Gass syndrome. Invest Ophthalmol Vis Sci 2013;54:3320-4. 